\title{
Lepton Universality Breaking vs Lepton Flavor Violation in Kaon Physics
}

\author{
Paride Paradisi* \\ Departament de Física Teòrica and IFIC, \\ Universitat de València-CSIC, E-46100 Burjassot, Spain E-mail: \\ paride.paradisi@uv.es
}

Deviations from the Standard Model (SM) expectations of the Lepton Universality (LU) breaking represent a powerful tool to probe New Physics (NP) effects. Kaon physics is an obvious ground where to perform such tests, for instance in the well studied $R_{K}^{e / \mu}=\mathscr{B}(K \rightarrow e v) / \mathscr{B}(K \rightarrow \mu v)$. Supersymmetric (SUSY) extensions of the Standard Model can exhibit $\mu-e$ non-universal contributions. Their origin is twofold: those deriving from lepton flavor conserving couplings are subdominant with respect to those arising from lepton flavor violating (LFV) sources. $\mu-e$ nonuniversality in $K_{\ell 2}$ is quite effective in constraining relevant regions of SUSY models with LFV. A comparison with analogous bounds coming from $\tau$ LFV decays shows the relevance of the measurement of $R_{K}^{e / \mu}$ to probe LFV in SUSY.

KAON International Conference

May 21-25 2007

Laboratori Nazionali di Frascati dell'INFN, Rome, Italy

\footnotetext{
* Speaker.
} 


\section{Introduction}

High precision electroweak tests, such as deviations from the SM expectations of the LFU breaking, represent a powerful tool to probe the SM and, hence, to constrain or obtain indirect hints of new physics beyond it. Kaon and pion physics are obvious grounds where to perform such tests, for instance in the $\pi \rightarrow \ell v_{\ell}$ and $K \rightarrow \ell v_{\ell}$ decays, where $\ell=e$ or $\mu$. In particular, the ratios

$$
R_{P}^{e / \mu}=1+\Delta r_{P}^{e / \mu}=\frac{\mathscr{B}(P \rightarrow \mu v)}{\mathscr{B}(P \rightarrow e v)}
$$

can be predicted with excellent accuracies in the SM, both for $P=\pi(0.02 \%$ accuracy [1] $)$ and $P=K(0.04 \%$ accuracy [1]), allowing for some of the most significant tests of LFU. These precision tests are equally interesting and fully complementary to the flavour-conserving electroweak precisions tests and to the FCNC tests performed at $B$ factories: the smallness of NP effects is more than compensated by the excellent experimental resolution and the good theoretical control.

The limiting factor in the determination $R_{K}^{e / \mu}$ is the $K \rightarrow e v$ rate, whose experimental knowledge has been quite poor so far.

The current world average $R_{K}^{e / \mu}=(2.45 \pm 0.11) \times 10^{-5}$ [ [†] will be soon improved thanks to a series of preliminary results by NA48/2 and KLOE (see Fig. 11). The two results by NA48/2, being based on different data sets (2003 [⿰氵月] and 2004 [3], respectively) with different running conditions, should be regarded as completely independent. Combining these new results with the PDG value yields [5]

$$
R_{K}^{e / \mu}=(2.457 \pm 0.032) \times 10^{-5} .
$$

This result is in good agreement with the SM expectation and has a relative error $(\sim 1.3 \%)$ three times smaller compared to the previous world average. Further improvements in the knowledge of $R_{K}^{e / \mu}$ would be more than welcome. Moreover, also the KLOE collaboration will reach an error down to the $1 \%$ level on $R_{K}^{e / \mu}$, once the remaining statistics will be added and the reconstruction efficiency improved [6].

Last bu not least, an error on $R_{K}^{e / \mu}$ of about $0.3 \%$ is the ambitious goal of the 2007 dedicated run of the CERN-P326 collaboration (the successor of NA48) [3, 7]. If these expectations will be fulfilled, in a short term the world average of $R_{K}^{e / \mu}$ will decrease by an additional factor of four.

In the following, we consider low-energy minimal SUSY extensions of the SM (MSSM) with $\mathrm{R}$ parity as the source of new physics to be tested by $R_{K}$ [8]. The question we intend to address is whether SUSY can cause deviations from $\mu-e$ universality in $K_{l 2}$ at a level which can be probed with the present attained experimental sensitivity, namely at the percent level. We will show that i) it is indeed possible for regions of the MSSM to obtain $\Delta r_{N P}^{e-\mu}$ of $\mathscr{O}\left(10^{-2}\right)$ and ii) such large contributions to $K_{\ell 2}$ do not arise from SUSY lepton flavor conserving (LFC) effects, but, rather, from LFV ones.

\section{Lepton Universality in $K \rightarrow \ell v$}

Due to the V-A structure of the weak interactions, the SM contributions to $K_{\ell 2}$ are helicity suppressed; hence, these processes are very sensitive to non-SM effects (such as multi-Higgs effects) which might induce an effective pseudoscalar hadronic weak density. In particular, charged 


\begin{tabular}{lc}
\hline \hline & $R_{K}^{e / \mu}\left[10^{-5}\right]$ \\
\hline PDG 2006 [⿰] & $2.45 \pm 0.11$ \\
NA48/2 '03 prel. [2] & $2.416 \pm 0.043 \pm 0.024$ \\
NA48/2 '04 prel. [3] & $2.455 \pm 0.045 \pm 0.041$ \\
KLOE prel. [6] & $2.55 \pm 0.05 \pm 0.05$ \\
\hline SM prediction & $2.472 \pm 0.001$ \\
\hline \hline
\end{tabular}

Figure 1: Current experimental data on $R_{K}^{e / \mu}$.

Higgs bosons $\left(H^{ \pm}\right)$appearing in any model with two Higgs doublets (including the SUSY case) can contribute at tree level to the above processes. The tree level partial width is given by [9]:

$$
\frac{\Gamma\left(K^{-} \rightarrow \ell^{-} \bar{v}\right)}{\Gamma\left(K^{-} \rightarrow \ell^{-} \bar{v}\right)_{S M}}=r_{K}=\left[1-\tan ^{2} \beta\left(\frac{m_{K}^{2}}{m_{H^{ \pm}}^{2}}\right)\right]^{2},
$$

Eq. (2.1) clearly show that a tree level contribution doesn't introduce any lepton flavour dependent correction. The first SUSY contributions violating the $\mu-e$ universality in $K \rightarrow \ell v$ decays arise at the one-loop level with various diagrams involving exchanges of (charged and neutral) Higgs scalars, charginos, neutralinos and sleptons. For our purpose, it is relevant to divide all such contributions into two classes: i) LFC contributions where the charged meson M decays without FCNC in the leptonic sector, i.e. $K \rightarrow \ell v_{\ell}$; ii) LFV contributions $K \rightarrow \ell_{i} v_{k}$, with $i$ and $k$ referring to different generations (in particular, the interesting case will be for $i=e, \mu$, and $k=\tau$ ).

\subsection{The lepton flavour conserving case}

One-loop corrections to $R_{K}$ include box, wave function renormalization and vertex contributions from SUSY particle exchange. The dominant diagrams containing one loop corrections to the $l W v_{l}$ vertex have the following suppression factors (compared to the tree level graph) [8]

$$
\Delta r_{K}^{e / \mu} \sim \frac{g_{2}^{2}}{16 \pi^{2}} \frac{m_{W}^{2}}{M_{S U S Y}^{2}}\left(\frac{m_{\tilde{\ell}_{\mu}}^{2}-m_{\tilde{\ell}_{e}}^{2}}{m_{\tilde{\ell}_{\mu}}^{2}+m_{\tilde{\ell}_{e}}^{2}}\right)
$$

for loops generated by charginos/neutralinos and sleptons. Even if we assume $\mathscr{O}(1)$ a quite large mass splitting among slepton masses we end up with $r_{K}^{e / \mu} \leq 10^{-4}$.

In conclusion, SUSY effects with flavor conservation in the leptonic sector can differently contribute to the $K \rightarrow e v_{e}$ and $K \rightarrow \mu v_{\mu}$ decays, hence inducing a $\mu-e$ non-universality in $R_{K}$, however such effects are still orders of magnitude below the level of the present experimental sensitivity on $R_{K}$. The same conclusions hold for $R_{\pi}$.

\subsection{The lepton flavour violating case}

It is well known that models containing at least two Higgs doublets generally allow flavour violating couplings of the Higgs bosons with the fermions. In the MSSM such LFV couplings are 
absent at tree level. However, once non holomorphic terms are generated by loop effects (so called HRS corrections [10]) and given a source of LFV among the sleptons, Higgs-mediated (radiatively induced) $H \ell_{i} \ell_{j}$ LFV couplings are unavoidable [11].

It has been shown [8] that Higgs-mediated LFV couplings generate a breaking of the $\mu-e$ universality in the purely leptonic $K^{ \pm}$decay.

One could naively think that SUSY effects in the LFV channels $K \rightarrow \ell_{i} v_{k}$ are further suppressed with respect to the LFC ones. On the contrary, charged Higgs mediated SUSY LFV contributions, in particular in the kaon decays into an electron or a muon and a tau neutrino, can be strongly enhanced.

The quantity which now accounts for the deviation from the $\mu-e$ universality reads:

$$
R_{K}^{L F V}=\frac{\sum_{i} \Gamma\left(K \rightarrow e v_{i}\right)}{\sum_{i} \Gamma\left(K \rightarrow \mu v_{i}\right)} \quad i=e, \mu, \tau .
$$

with the sum extended over all (anti)neutrino flavors (experimentally one determines only the charged lepton flavor in the decay products).

The dominant SUSY contributions to $R_{K}^{L F V}$ arise from the charged Higgs exchange. The effective LFV Yukawa couplings we consider are

$$
\ell H^{ \pm} v_{\tau} \rightarrow \frac{g_{2}}{\sqrt{2}} \frac{m_{\tau}}{M_{W}} \Delta_{R}^{3 l} \tan ^{2} \beta \quad \ell=e, \mu .
$$

A crucial ingredient for the effects we are going to discuss is the quadratic dependence on $\tan \beta$ in the above coupling: one power of $\tan \beta$ comes from the trilinear scalar coupling in Fig.1, while the second one is a specific feature of the above HRS mechanism.

The LFV $\Delta_{R}^{3 \ell}$ parameters are induced at one loop level and it turns out that $\Delta_{R}^{3 \ell} \leq 10^{-3}$ [ 8$]$. Making use of the LFV Yukawa coupling in Eq. (2.3), it turns out that the dominant contribution to $\Delta r_{N P}^{e-\mu}$ reads [8]:

$$
R_{K}^{L F V} \simeq R_{K}^{S M}\left[1+\left(\frac{m_{K}^{4}}{M_{H}^{4}}\right)\left(\frac{m_{\tau}^{2}}{m_{e}^{2}}\right)\left|\Delta_{R}^{31}\right|^{2} \tan ^{6} \beta\right] .
$$

In Eq. (2.4) terms proportional to $\Delta_{R}^{32}$ are neglected given that they are suppressed by a factor $m_{e}^{2} / m_{\mu}^{2}$ with respect to the term proportional to $\Delta_{R}^{31}$.

Taking $\Delta_{R}^{31} \simeq 5 \cdot 10^{-4}, \tan \beta=40$ and $M_{H}=500 \mathrm{GeV}$ we end up with $R_{K}^{L F V} \simeq R_{K}^{S M}(1+0.013)$. We see that in the large (but not extreme) $\tan \beta$ regime and with a relatively heavy $H^{ \pm}$, it is possible to reach contributions to $\Delta r_{K S U S Y}^{e-\mu}$ at the percent level thanks to the possible LFV enhancements arising in SUSY models.

Turning to pion physics, one could wonder whether the analogous quantity $\Delta r_{\pi S U S Y}^{e-\mu}$ is able to constrain SUSY LFV. However, the correlation between $\Delta r_{\pi S U S Y}^{e-\mu}$ and $\Delta r_{K S U S Y}^{e-\mu}$ :

$$
\Delta r_{\pi S U S Y}^{e-\mu} \simeq\left(\frac{m_{d}}{m_{u}+m_{d}}\right)^{2}\left(\frac{m_{\pi}^{4}}{m_{k}^{4}}\right) \Delta r_{K S U S Y}^{e-\mu}
$$

clearly shows that the constraints on $\Delta r_{K s u s y}^{e-\mu}$ force $\Delta r_{\pi s u s y}^{e-\mu}$ to be much below its actual experimental upper bound.

A key ingredient for the generation of LFU breaking effects are large $\tan \beta$ values so, it is legitimate to ask how natural this framework is. 
In this respect, we remind that values of $\tan \beta \sim 30-50$ can allow the unification of top and bottom Yukawa couplings, as predicted in well-motivated grand-unified models [12]. Moreover, from a low energy point of view, large $\tan \beta$ values lead to interesting phenomenological virtues [13]: the present $(g-2)_{\mu}$ anomaly and the upper bound on the Higgs boson mass can be easily accommodated, while satisfying all the present tight constraints in the electroweak and flavor sectors. Additional low-energy signatures of this scenario could possibly show up in the near future in $\mathscr{B}(B \rightarrow \tau v)$ and $\mathscr{B}\left(B_{s, d} \rightarrow \ell^{+} \ell^{-}\right)$. Additionally, in the regime with large $\tan \beta$, the relic-density constraints can be easily satisfied mainly in the so called $A$-funnel region [14].

\section{References}

[1] W.J. Marciano and A. Sirlin, Phys.Rev.Lett. 713629 (1993); M.Finkemeier, Phys.Lett. B 387391 (1996); V. Cirigliano and I. Rosell, arXiv:0707.3439 [hep-ph], arXiv:0707.4464 [hep-ph].

[2] L. Fiorini, PoS HEP2005, 288 (2006).

[3] V. Kozhuharov, these proceedings.

[4] W. M. Yao et al. [Particle Data Group], J. Phys. G 33 (2006) 1.

[5] R. Wanke, these proceedings, arXiv:0707.2289 [hep-ex].

[6] A. Sibidanov, these proceedings.

[7] G. Ruggiero, these proceedings.

[8] A. Masiero, P. Paradisi, and R. Petronzio Phys. Rev. D 74, 011701 (2006) [arXiv:hep-ph/0511289].

[9] W.S.Hou, Phys. Rev. D 48, 2342 (1992).

[10] L. J. Hall, R. Rattazzi and U. Sarid, Phys. Rev. D 50, 7048 (1994).

[11] K. S. Babu and C. Kolda, Phys. Rev. Lett. 89 (2002) 241802 [hep-ph/0206310].

[12] G. Anderson, S. Raby, S. Dimopoulos, L. J. Hall and G. D. Starkman, Phys. Rev. D 49 (1994) 3660 [hep-ph/9308333].

[13] G. Isidori and P. Paradisi, Phys. Lett. B 639 (2006) 499 [hep-ph/0605012].

[14] J. R. Ellis, L. Roszkowski and Z. Lalak, Phys. Lett. B 245 (1990) 545. 